\title{
Mini-symposium
}

\section{Clinical significance of patent foramen ovale: introduction}

$\mathrm{S}$ ince Lechat's and Webster's seminal reports, the statistical association of cryptogenic stroke and patent foramen ovale (PFO) has intrigued both cardiologists and neurologists. Growing technical experience and improved percutaneous devices have encouraged cardiologists increasingly to close PFOs. Nevertheless, the indication for this procedure often remains problematic. Clearly, paradoxical embolism through a PFO is a well recognised complication of deep venous thrombosis, especially in the context of pulmonary embolism with its attending increase in right sided pressures. This scenario, however, is relatively rare. In contrast, the true importance of paradoxical embolism through a PFO in other circumstances remains unclear.

In this mini-symposium, the importance of PFO and the "case for closure" is expertly reviewed from different angles. Fausto Pinto discusses the echocardiographic diagnosis of patent foramen ovale; Pierre Amarenco reviews the observational basis for the imputed causality between PFO and cerebral ischaemia; and Bernhard Meier explains the interventional closure procedure and the devices.

F A Flachskampf, W G Daniel Medizinische Klinik II, Universitätsklinik Erlangen, Germany

Correspondence to: Professor Frank A Flachskampf; frank.flachskampf@ med2.med.uni-erlangen.de

\section{IMAGES IN CARDIOLOGY}

\section{Crab claw-like appearance on coronary angiography}

A 61 year old man, undergoing dialysis because of chronic renal failure, was admitted for examination with high grade fever. A blood sample was positive for methicillin resistant Staphylococcus aureus. During his stay in hospital, he complained of acute chest pain associated with ST elevation in leads II, III, aVF. Transthoracic echocardiography (TTE) showed an akinetic inferior wall and a smooth bulging mass located along the atrioventricular groove (panel A). Colour Doppler imaging revealed blood flow from the bulging mass into the right atrium (panel B). No vegetations were noted on TTE. Coronary angiography demonstrated a large aneurysm of the right coronary artery, which was occluded by thrombus (panel C). Contrast media injected into the right coronary artery drained to the right atrium (panel D). The patient underwent resection of the aneurysm and patch closure of the perforation following removal of the aneurysm. Histological examination showed it was a mycotic aneurysm.

The patient's postoperative course was uneventful, and he was discharged from hospital and placed on a long course of antibiotics.

Coronary artery mycotic aneurysms are often silent until either thrombosis results in myocardial infarction or the aneurysm ruptures producing massive haemopericardium and cardiac tamponade. Given the rarity of the condition,
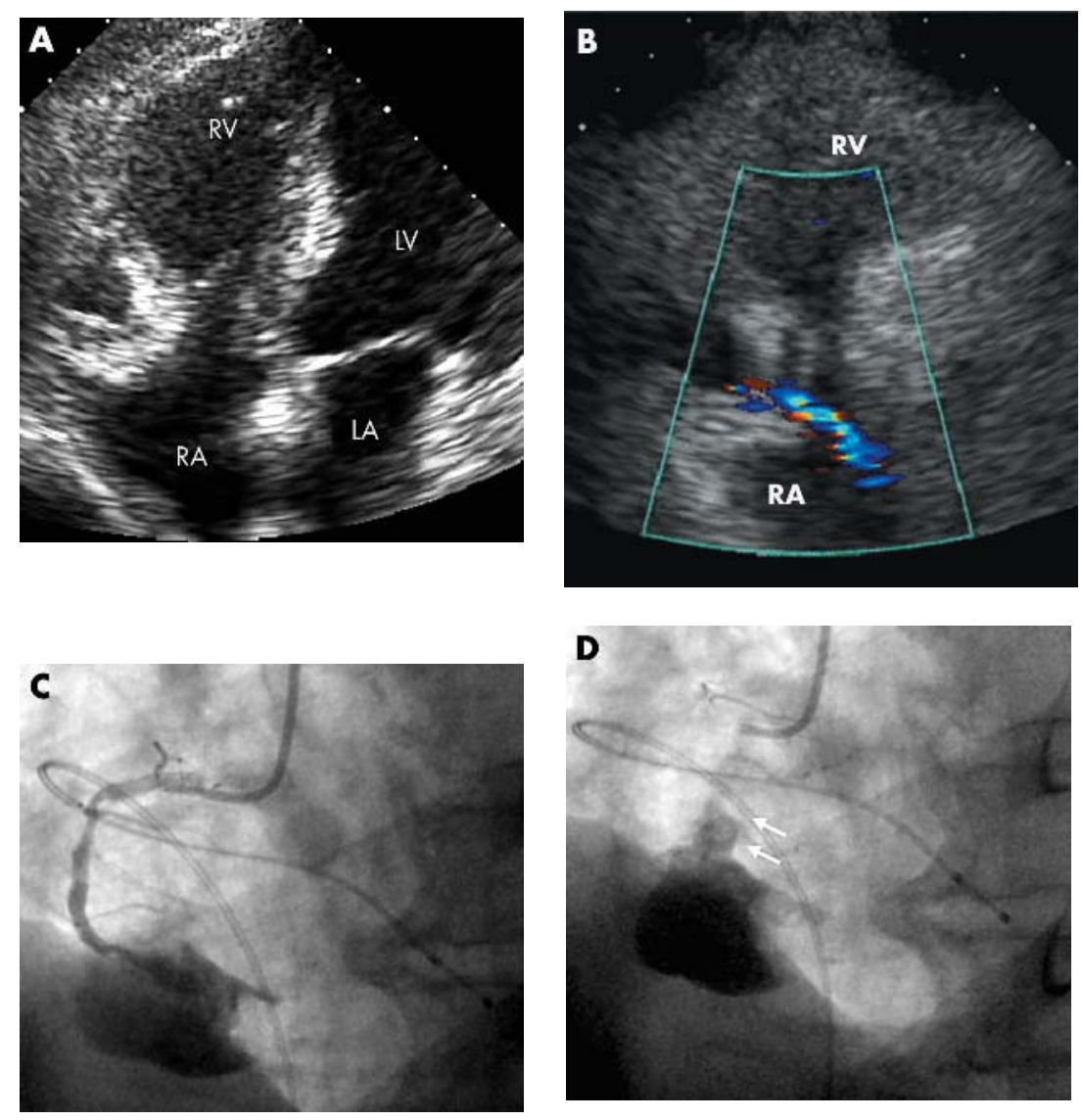

an extremely high index of clinical $\mathrm{HOe}$

$S$ Ehara J Yoshikawa suspicion is necessary for the diagnosis. 